\title{
Pneumatic Lithotripsy Versus Laser Lithotripsy for Ureteral Stones
}

\author{
Amir Reza Abedi ${ }^{1}$, Mohammad Reza Razzaghi ${ }^{1}$, Farzad Allameh' ${ }^{1}$, Fereshte Aliakbari², Morteza Fallah- \\ Karkan $^{3 *}$, Arash Ranjbar ${ }^{3}$
}

\begin{abstract}
${ }^{1}$ Laser Application in Medical Science Research Center, Shahid Beheshti University of Medical Sciences, Tehran, Iran ${ }^{2}$ Infertility \& Reproductive Health Research Center, Shahid Beheshti University of Medical Sciences, Tehran, Iran ${ }^{3}$ Shohada-e-Tajrish Hospital, Shahid Beheshti University of Medical Sciences, Tehran, Iran
\end{abstract}

\author{
*Correspondence to \\ Morteza Fallah-Karkan, Shohada- \\ e-Tajrish hospital, Shahid Beheshti \\ University of Medical Sciences, \\ Tehran, Iran. \\ Tel: +98 9111863352 \\ Fax: +982122712234 \\ Email: Mortezafallah.md@gmail.com
}

Published online 17 September 2018

\begin{abstract}
Introduction: Several different modalities are available for ureteral stone fragmentation. From them pneumatic and holmium: yttrium-aluminum-garnet (Ho: YAG) lithotripsy have supportive outcomes. In this study we studied 250 subjects who had ureteroscopic pneumatic lithotripsy (PL) or laser lithotripsy (LL).

Methods: Two-hundred fifty patients with ureteral stones underwent ureteroscopic lithotripsy (115 subjects in the PL group, 135 subjects in the LL group) from August 2010 to April 2016. The purpose of this investigation was to evaluate stone-free rate (SFR), mean operation time (MOT), mean hospital stay (MHS), stone migration and complications.

Results: Two groups were similar in age, gender, mean size of stones, side of stone, and complications. There was a statistical difference in terms of SFR, stone migration and MHS in favor of the LL group $(P \leq 0.05, P \leq 0.05$ respectively), and MOT in favor of the PL group $(P \leq$ 0.05).

Conclusion: Both the PL and LL techniques were effective and safe for ureteral stones, however a slightly higher SFR was found in the LL group.

Keywords: Pneumatic lithotripsy; Laser lithotripsy; Ureteral stone; Ho: YAG laser.
\end{abstract}

\section{Introduction}

Treating patients with urolithiasis is a part of daily urological practice ${ }^{1,2}$ and there are different treatment methods for ureteral calculi such as open stone surgery, extracorporeal shock wave lithotripsy (ESWL), percutaneous nephrolithotomy, laparoscopic ureterolithotomy, and ureteroscopic procedures. ${ }^{3}$

In the early 1980s open surgery was the best treatment for ureteral stones; but introducing the small caliber ureteroscope and ESWL resulted in the virtually extinction of open surgery. ${ }^{1}$ The main benefit of ureteroscopic surgery is visualization of the ureter that enables detection and treatment of ureteral stones. ${ }^{4}$

There is a variety of modalities for stone fragmentation including ultrasonic, electrohydraulic, pneumatic lithotripsy (PL) or laser lithotripsy (LL). ${ }^{5}$ A number of lasers have been trialed for the LL method, that the holmium: yttrium-aluminum-garnet (Ho: YAG) was the most commonly used treatment. ${ }^{6}$ Ho: YAG laser which mostly applied in stone fragmentation procedure is a pulsed mode modality with 2100 wavelength $(\mathrm{nm})$ and $\approx 0.5 \mathrm{~mm}$ tissue penetration characteristics. ${ }^{7}$

Both PL and Ho: YAG lithotripsy have favorable outcomes. ${ }^{8,9}$ The Swiss Lithoclast fragments the stones by oscillatory movements of metal probe against the stones. ${ }^{10}$ The thermal effect produced by Ho: YAG laser pulses are owing to creation of microscopic vaporization bubbles. The rapid implosion of the bubble at the tip of the fiber creates a shock wave which breaks the stones. ${ }^{11}$

In this study we studied 250 patients who had ureteroscopic PL or LL from 2010 to 2016 in order to compare the safety and efficacy of the two methods.

\section{Methods}

Study Population

This retrospective study included 250 out of 337 patients with ureteral stone who underwent ureteroscopic lithotripsy (the PL and LL treatment were done in 115 and 135 cases retrospectively according to the surgeonspatients decisions) in Shohade-e-Tajrish hospital, Shahid Beheshti University of Medical Sciences, Tehran, Iran from August 2010 to April 2016. Furthermore, Patients with renal abnormally, pelvic or caliceal stone, uncontrolled coagulopathy, severe musculoskeletal deformity and pregnant women were excluded from our study as well. Urine culture and renal function test and abdominopelvic 
non-contrast computed tomography scan (NCCTS) were done for all patients before procedure.

\section{Surgical Technique}

A single dose prophylactic intravenous antibiotic was administered before surgery. All the patients underwent spinal anesthesia and were then placed in lithotomy position. The ureteroscopic procedures were done by 5 surgeons in Shohade-e-Tajrish hospital urology department.

Ureteroscopic procedures were done using a 9.5 Fr (Wolf Inc., Germany) semirigid ureteroscope under direct endoscopic vision. Ureters were accessed via 0.035 inch guide wires.

For the PL group the $0.8 \mathrm{~mm}$ to $1.2 \mathrm{~mm}$ lithoclast was passed through the ureteroscope working channel. The tip of probe was rested on the stone surface and the probe was activated under $2.5 \mathrm{~atm}$ in either continues pulse or single shut mode.

In the LL group when stone was visible fragmented using Ho-YAG laser (Iranian National Laser Center, Iran); the laser fiber $(200 \mu \mathrm{m})$ was passed through working channel of ureteroscope to the surface of calculi. During the lithotripsy, laser was set to a power of 5-10 W and a frequency of $8-10 \mathrm{~Hz}$.

The flexible ureteroscope (8.5/5.3 Fr Flexible ureteroscope, Olympus) was used if it was necessary.

The stone was broken to particles less than $3 \mathrm{~mm}$ to increase the likelihood of spontaneous passage. Double J stent was inserted routinely after the procedure and the stent was removed in 2 weeks after the procedure in which the stone free status was achieved.

We defined stone-free rate (SFR) as the absence of particles $<3 \mathrm{~mm}$ in postoperative kidney, ureter, bladder (KUB) radiography in radiopaque calculi and NCCTS in radiolucent stone (64 patients).

Data Analysis

All data were evaluated by the statistical package for social sciences software (Chicago, IL, USA, version 18.0). Descriptive statistics (mean \pm standard deviation) and Student's $t$ test was used to show and analyze the quantitative outcomes. The qualitative data was presented with frequency and percentage and their analysis was done with the Fisher exact test and chi-square test. The $P$ values less than 0.05 were considered statistically significant.

\section{Results}

Table 1 illustrates the distribution of demographic and clinical characteristics among the two groups. Groups were similar as stated in age, gender, mean size of stones, and side of stone. Upper ureter stone site were significantly more in the LL groups.

As can be seen statistically significant differences were found in terms of SFR, stone migration and mean hospital stay (MHS) in favor of the LL group $(P \leq 0.05, P \leq 0.05$ respectively), and MOT in favor of the PL group ( $P \leq$ $0.05)$. The disadvantages of the LL group overshadowed its effectiveness in treatment of proximal ureteral calculi. The details of operation and postoperative data are delineated in Table 2.

Most patients in the PL group in who fragments were pushed back, had ureteral stone larger than $1.5 \mathrm{~cm}$ or stone embedded in ureteral edema. Seven patients were managed by flexible ureteroscopy and LL in the same session and 33 patients were treated with ESWL before removal of double J stent. In the LL arm, the particles were retropulsed to kidney in nine cases, in whom fragments were successfully fragmented in the renal pelvis or calyx by using flexible ureteroscope for seven and ESWL for two patients as well. Ureteroscopic access to stone was not possible in 2 patients.

Ureteral perforation was successfully managed by placing double J stent for six weeks. Only one patient in the PL group needed open surgery for a ureteral perforation.

The long-term complication was ureteral stenosis that was seen in two patients in the LL and one patient in the PL

Table 1. Demographic and Clinical Characteristics

\begin{tabular}{lcc}
\hline Variable & LL (n=135) & PL (n=115 ) \\
\hline Mean age \pm SD, y & $40.1 \pm 3.8$ & $39.2 \pm 4.3$ \\
Male, No. (\%) & $91(67.4)$ & $73(63.4)$ \\
Previous history of TUL, No. (\%) & $13(9.6)$ & $11(9.5)$ \\
Stone laterality & & \\
$\quad$ Right side, No. (\%) & $82(60.7)$ & $74(64.3)$ \\
$\quad$ Bilateral, No. (\%) & $6(4.4)$ & $7(6)$ \\
Stone location & $33(24.4)$ & $16(13.9)$ \\
$\quad$ Upper, No. $(\%)$ & $28(20.7)$ & $34(29.5)$ \\
$\quad$ Middle, No. (\%) & $74(54.8)$ & $65(56.5)$ \\
$\quad$ Distal, No. (\%) & $9.6 \pm 2.4(8-16)$ & $9.2 \pm 2.1(7-14)$ \\
Stone diameter, mm & $1.2 \pm 0.4$ & $1.2 \pm 0.1$ \\
Number of stones, $\mathrm{n}$ & & $96(83.4)$ \\
Duration of stone impaction & $109(80.7)$ & $19(16.5)$ \\
\hline$<2$ months, No. (\%) & $26(19.2)$ & \\
\hline
\end{tabular}

Abbreviation: TUL, transurethral lithotripsy. $P \geq 0.05$.

Table 2. Operative and Postoperative Data

\begin{tabular}{llll}
\hline Variable & LL $(\mathbf{n = 1 3 5})$ & PL $(\mathbf{n = 1 1 5})$ & $\boldsymbol{P}$ \\
\hline Complications & & & $\geq 0.05$ \\
$\quad$ Ureteral perforation, No. $(\%)$ & $1(0.7)$ & $5(4.5)$ & \\
Postoperative fever, No. (\%) & $1(0.7)$ & $3(2.6)$ & \\
Mucosal damage, No. (\%) & $4(2.9)$ & $6(5.2)$ & \\
MOT \pm SD, min & $14.4 \pm 2.05$ & $10.01 \pm 6.2$ & $\leq 0.05$ \\
MHS \pm SD, h & $25.04 \pm 2.1$ & $26.2 \pm 0.8$ & $\leq 0.05$ \\
Immediate stone-free status, & $126(93.3)$ & $75(65.2)$ & $\leq 0.05$ \\
No. (\%) & & & \\
Stone migration, No. (\%) & $40(29.6)$ & $9(7.8)$ & $\leq 0.05$ \\
\hline
\end{tabular}

Abbreviations: MOT, Mean operation time; MHS, Mean hospital stay 
group and was managed successfully by laser ureterotomy and placing double J stent.

\section{Discussion}

Recent improvement in equipment and technologies made great strides in the management of patients with urinary calculi. ${ }^{12}$ In the current era, minimally invasive treatments are used for ureteral calculi. One of these minimally invasive approaches is PL which has benefits, such as less expensiveness and safety. It also has some complications, for instance stone migration. ${ }^{13}$ The other approach is Ho: YAG laser which is one of the safest, most effective, and most adaptable lithotripters. Further benefits of the holmium laser include its significantly smaller postlithotripsy particles compared with other lithotripters. The Ho: YAG laser produces a weak shockwave, which decreases the likelihood of push back of the calculi or stone fragments. ${ }^{3}$ Ho: YAG LL is a reliable method of stone fragmentation regardless of the stone hardness and composition, and it can be conducted through all types of ureteroscope. ${ }^{14}$

In the present study, comparison between 2 types of lithotripsy revealed that in terms of SFR, MHS and retreatment rate, laser lithotripsy has advantages over PL since stone fragments are less likely to migrate in LL group.

Devarajan et $\mathrm{al}^{15}$ reported a $90 \%$ success rate in 300 lithotripsy patients in whom a holmium laser was used with complications observed in 10 patients. Strictures were more commonly seen with impacted calculi in the upper ureter early in the series. Jeon et $\mathrm{al}^{16}$ showed SFR of $96 \%$ for a Ho: YAG laser arm and $37.1 \%$ in the lithoclast arm $(P<0.05)$. Considering MOT, Ho: YAG laser lithotripsy also had advantages over the PL method. The time taken for stones to be fragmented into removable volume might be shorter in PL but usually particles were too large to pass spontaneously and it was necessary to use basket or grasper to remove these fragments. The results of the mentioned studies were in accordance with our results.

In comparison with lithotripsy, Ho: YAG laser was associated with milder injury to the ureter because of superficial penetration depth of laser. MHS in the LL group was significantly shorter than the PL group due to milder injury to ureter in the LL group. The finding of our study was in line with the preceding investigations in the literature about efficacy of ureteroscopic Ho: YAG laser lithotripter. ${ }^{17-20}$

Long duration of ureteral stone (more than 3 months) was related to the presence of a ureteral polyp. Polyps impeded ureteroscopic access in the PL group, but 3 polyps can be treated with laser in the LL group so making ureteroscopic access possible in most of the cases. ${ }^{17}$

This study has several limitations, the procedures were done by surgeons with different experience that might lead to bias in our results, in addition our investigation was retrospective study and we recommend conducting prospective randomized study to confirm our results.

\section{Conclusion}

In conclusion, we found that both the PL and LL approaches were effective and safe for ureteral stone, but the LL method had advantages, especially in stone free rate, over the PL treatment. Another advantage of the LL method was safe stone fragmentation in upper ureteral calculi due to lower push back rate in contrast with the PL method.

\section{Ethical Considerations}

Researchers undertook to do the research based on the approval of the ethical committee of Shahid Beheshti University of Medical Sciences.

\section{Conflict of Interests}

This investigation has no conflict of interest to declare by any author.

\section{Acknowledgments}

The authors wish to acknowledge the assistance of Urology staff.

\section{References}

1. Rosa M, Usai P, Miano R, et al. Recent finding and new technologies in nephrolithiasis: a review of the recent literature. BMC Urol. 2013;13:10. doi:10.1186/1471-249013-10

2. Javanmard B, Fallah Karkan M, Razzaghi MR, Ghiasy S, Ranjbar A, Rahavian A. Surgical management of vesical stones in children: a comparison between open cystolithotomy, percutaneous cystolithotomy and transurethral cystolithotripsy with holmium-YAG Laser. J Lasers Med Sci. 2018;9(3):183-187. doi:10.15171/ jlms.2018.33

3. Lingeman JE. Surgical management of upper urinary tract calculi. In: Kavoussi LR, Novick AC, Partin AW, Peters CA, Wein AJ, eds. Campbell-Walsh Urology. Philadelphia: Saunders-Elsevier; 2006:1431-1507.

4. Abedi AR, Allameh F, Razzaghi MR, et al. The Efficacy and Safety of Laser Lithotripsy in Pregnancy. J Lasers Med Sci. 2017;8(2):84-87. doi:10.15171/jlms.2017.15

5. Fallah Karkan M, Ghiasy S, Ranjbar A, Javanmard B. Evaluation of $200 \mathrm{Mm}, 365 \mathrm{Mm}$ and $500 \mathrm{Mm}$ fibers of Ho:YAG laser in transurethral lithotripsy of ureteral: a randomize control trial. J Lasers Med Sci. 2018;9(1):69-72. doi:10.15171/jlms.2018.14

6. Razzaghi MR, Fallah Karkan M, Ghiasy S, Javanmard B. Laser application in iran urology: a narrative review. J Lasers Med Sci. 2018;9(1):1-6. doi:10.15171/jlms.2018.01

7. Floratos DL, de la Rosette JJ. Lasers in urology. BJU Int. 1999;84(2):204-211.

8. Denstedt JD, Razvi HA, Rowe E, Grignon DJ, Eberwein PM. Investigation of the tissue effects of a new device for intracorporeal lithotripsy--the Swiss Lithoclast. J Urol. 1995;153(2):535-537. doi:10.1097/00005392-19950200000078 
9. Denstedt JD, Eberwein PM, Singh RR. The Swiss Lithoclast: a new device for intracorporeal lithotripsy. $J$ Urol. 1992;148(3 Pt 2):1088-1090.

10. Cecchetti W, Zattoni F, Nigro F, Tasca A. Plasma bubble formation induced by holmium laser: an in vitro study. Urology. 2004;63(3):586-590. doi:10.1016/j. urology.2003.09.010

11. Razzaghi MR, Razi A, Mazloomfard MM, Mokhtarpour H, Javanmard B, Mohammadi R. Trans-Ureteral Ureterolithotripsy of Ureteral Calculi:Which is the Best; Pneumatic or Holmium Laser Technique? J Lasers Med Sci. 2011;2(2):59-62. doi:10.22037/2010.v2i2.2282

12. Javanmard B, Razaghi MR, Ansari Jafari A, Mazloomfard MM. Flexible Ureterorenoscopy Versus Extracorporeal Shock Wave Lithotripsy for the Treatment of Renal Pelvis Stones of 10-20 mm in Obese Patients. J Lasers Med Sci. 2015;6(4):162-166. doi:10.15171/jlms.2015.12

13. Razaghi MR, Razi A. Comparison between the Holmium Laser (Made in Iran) and Pneumatic Lithotripsy in Patients Suffering from Upper Ureteral Stone between $1-2 \mathrm{~cm}$. J Lasers Med Sci. 2012;2(4):144-147. doi:10.22037/2010. v2i4.2623

14. Cui Y, Cao W, Shen H, et al. Comparison of ESWL and ureteroscopic holmium laser lithotripsy in management of ureteral stones. PLoS One. 2014;9(2):e87634. doi:10.1371/ journal.pone.0087634

15. Devarajan R, Ashraf M, Beck RO, Lemberger RJ, Taylor MC. Holmium: YAG lasertripsy for ureteric calculi: an experience of 300 procedures. Br J Urol. 1998;82(3):342347.

16. Jeon SS, Lee KS, Won HS, et al. A comparison of Holmium: YAG laser with lithoclast lithotripsy in ureteral calculi fragmentation. Korean J Urol. 2000;41(3):375-380.

17. Razzaghi MR, Razi A, Mazloomfard MM, Golmohammadi Taklimi A, Valipour R, Razzaghi Z. Safety and efficacy of pneumatic lithotripters versus holmium laser in management of ureteral calculi: a randomized clinical trial. Urol J. 2013;10(1):762-766.

18. Garg S, Mandal AK, Singh SK, et al. Ureteroscopic laser lithotripsy versus ballistic lithotripsy for treatment of ureteric stones: a prospective comparative study. Urol Int. 2009;82(3):341-345. doi:10.1159/000209369

19. Kostakopoulos A, Stavropoulos NJ, Picramenos D, Kyriazis P, Deliveliotis C. The Swiss lithoclast: an ideal intracorporeal lithotripter. Urol Int. 1995;55(1):19-20. doi: $10.1159 / 000282740$

20. Yip KH, Lee CW, Tam PC. Holmium laser lithotripsy for ureteral calculi: an outpatient procedure. J Endourol. 1998;12(3):241-246. doi:10.1089/end.1998.12.241 